\title{
TVE, su legislación y su afectación en los Centros Territoriales
}

TVE, its legislation and its impact on the Territorial Centres

Marta Pérez Ruiz, Universidad de Burgos- martapr@ubu.es

\section{Resumen}

La legislación que ha afectado a Televisión Española sí ha regulado cómo debía ser la estructura de la televisión pública en este país pero, en ningún caso, ha especificado cómo debía ser su programación o cuál debía ser la duración de las desconexiones territoriales para cumplir con las funciones de servicio público que indicaba esta misma literatura legal. Desde el Estatuto de 1980 hasta la Ley 17/2006, de 5 de junio, nos encontramos con que hay poca regulación, más allá de las directrices marcadas por la dirección de TVE, para el desarrollo de las delegaciones de TVE.

Palabras clave

Legislación, TVE, delegaciones, desconexiones.

Abstract

The legislation has affected Spanish Television itself has regulated how it should be the structure of public television in this country but in no case has specified how it should be your schedule, or what should be the duration of the territorial disconnections to meet public service functions indicating the same legal literature. From 1980 to Statute Law 17/2006 of June 5th , we find that there is little regulation beyond the guidelines set by the management of TVE, for the development of the delegations of TVE.

\section{Keywords}

Legislation, TVE, delegationes, disconnections.

Sumario

1. Introducción. 2. Metodología. 3. Resultado. 4. Conclusiones. 5. Bibliografía. 


\section{Introducción}

Desde el nacimiento de Televisión Española ha sido numerosa la legislación que ha afectado a esta televisión pública. Pero no ha habido muchos estudios sobre esta misma legislación, sobre todo, en lo que se refiere a cómo ha podido afectar a los Centros Territoriales que forman parte de la estructura de esta cadena de televisión. A la hora de realizar el análisis de las leyes que han afectado y afectan a TVE en cuanto a su funcionamiento y organización, nos vamos a concentrar en el siguiente periodo de análisis, 1982-2012. Nos circunscribimos a estos años porque es cuando se desarrolla el mayor proceso legislativo en este aspecto y porque, es a partir de 1982, cuando se termina de configurar la red de delegaciones territoriales y éstas se pueden ver afectadas por las distintas leyes y reglamentos. El estudio termina en el año 2012 porque es un año en el que la producción de los Centros Territoriales de TVE se vio muy limitada y, por lo tanto, también su desarrollo, al mismo tiempo que no se plantearon nuevas alternativas para estas delegaciones.

A lo largo de este recorrido por la legislación, nos vamos a centrar en las cuestiones relativas a los Centros Territoriales. Consideramos que ya existen análisis, que nos han servido de referencia, sobre esta legislación, pero por nuestra temática hemos elegido aquellas cuestiones que son más cercanas a la organización territorial de TVE y a la normativa que se debe aplicar. En este sentido, incluimos aspectos relativos a los Consejos Asesores de RTVE en las Comunidades Autónomas y a la Comisión Mixta de Control de RTVE, formada por parlamentarios del Congreso de los Diputados y del Senado ante la que también se han expuesto cuestiones relacionadas con el control y situación de estos Centros Territoriales.

A este razonamiento tenemos que añadir que no es hasta 1980, año en el que se promulga el Estatuto de Radio y Televisión cuando se puede asegurar que existen indicaciones para el desarrollo territorial de TVE en todo la geografía española, aunque veremos también que existen disposiciones y artículos que no se llegaron a convertir en realidad, no por voluntad del legislador, sino por condiciones externas, como la puesta en funcionamiento de un Tercer Canal por parte de TVE para la emisión de contenidos de las comunidades autónomas.

\section{Metodología}

A la hora de realizar este planteamiento de análisis, el abordaje se ha realizado desde la consulta de la bibliografía previa existente y en el estudio de toda la legislación existente y en la que se pudiera ver afectada la estructura territorial de Televisión Española. Este análisis se ha hecho partiendo del estudio de toda la legislación más relevante dese 1982 hasta 2012. En el desarrollo de este artículo realizaremos una mención especial al informe del denominado Comité de sabios ${ }^{1}$ que elaboró un análisis en profundidad por la situación de RTVE, incluyendo cuál debería ser el papel a desempeñar por los Centros Territoriales de TVE en las distintas comunidades autónomas como promotoras, además, de la industria audiovisual de cada región.

Este trabajo de estudio de la legislación que afecta a TVE en nuestro periodo de estudio incluye también las leyes promulgadas en los años 2006 y 2010 que afectan, la primera de RTVE y la segunda a todo el sistema audiovisual. No nos olvidamos de incluir el Real Decreto de 20 de abril de 2012 y que afecta directamente a la elección del presidente de la Corporación RTVE.

\section{Resultado}

\subsection{Estatuto de Radio y Televisión $1980^{2}$}

Antes de comenzar el análisis de este Estatuto, cabe recordar que la Constitución Española de 1978 recoge en el artículo 20.1d el derecho: "A comunicar o recibir libremente información veraz por cualquier medio de difusión". A esto hay que añadir que, en este mismo artículo, se indica que:

La ley regulará la organización y el control parlamentario de los medios de comunicación social dependientes del Estado o de cualquier ente público y garantizará el acceso a dichos medios de los grupos sociales y políticos significativos, respetando el pluralismo de la sociedad y de las diversas lenguas de España.

Por lo tanto, el legislador a la hora de elaborar este Estatuto debía regular en función del mandato que había recogido de la Constitución. De esta manera, lo primero que se recoge en este texto legal es que la radiodifusión y la televisión son servicios públicos esenciales cuya titularidad es del Estado, al mismo tiempo que se contempla la posibilidad de ceder a las Comunidades Autónomas la gestión directa de un canal de televisión de titularidad estatal que se cree para ese determinado ámbito estatal3. Dentro de la definición de los ejes en los que se debía basar RTVE, el Estatuto recoge, en el artículo quinto, que la gestión directa de los servicios públicos de radio y televisión se ejercerá a través de RTVE a la que se le encomienda el desarrollo de la función de servicio público entendida como la producción y emisión de un

\footnotetext{
${ }^{1}$ El Consejo para la Reforma de los medios de comunicación de titularidad del Estado se creó por Real Decreto 744/2004 del 23 de abril de 2004. Este Consejo, también denominado Comité de Sabios estaba compuesto por Emilio Lledó, Enrique Bustamante, Victoria Camps, Fernando Fernández Sabater, Fernando González Urbaneja, que entró en el Consejo como sustituto tras la muerte de Fernando Lázaro Carreter. El experto en materia de financiación fue Miguel Ángel Arnedo.

2 Según recoge Leopoldo Abad Alcalá en El Servicio Público de Televisión ante el siglo XXI, 1999, citando a Carreras Serra, LL., 1996, el Estatuto de Radio y Televisión se constituyó como la norma básica sobre la televisión en España, aunque solo los primeros cuatro artículos se refieren a la radiodifusión en general, ya que a partir del artículo 5, esta Ley regula el Ente Público RTVE y sus empresas filiales.

${ }^{3}$ Capítulo I, artículo 2.2 Estatuto Radio y Televisión 1980.
} 
Una vez que quedan definidos los conceptos de servicio público y cuáles deben ser las funciones que debe desempeñar RTVE para cumplirlo, en la Sección VI encontramos los artículos que se refieren a la organización territorial del Ente. De esta manera, el artículo 13 de este Estatuto indica que RTVE deberá elaborar una propuesta de programación tanto de radio como de televisión para que pueda ser emitida en el ámbito territorial en función de los porcentajes que se hayan establecido sin perjudicar a la programación nacional.

En el artículo 14 se estipula que en cada Comunidad Autónoma tiene que existir un Delegado Territorial de RTVE que será nombrado por el Director General de la entidad, una vez que se haya tomado en consideración al órgano representativo que se constituya en cada territorio. En este caso, este artículo se refiere a la presencia de los Consejos Asesores que se indican en el punto dos de este mismo artículo y que deberá ser creado por el propio gobierno de la Comunidad Autónoma ${ }^{5}$. En el artículo quince se indica que el Delegado territorial será quien se encargue de elevar al Director general de RTVE una propuesta de programación así como sus horarios de emisión en el ámbito territorial, una vez que esta propuesta se haya presentado ante el Consejo asesor autonómico.

Respecto a las funciones del Consejo Asesor de RTVE cada Comunidad Autónoma legisló las funciones y composición de cada órgano. Si tomamos, por ejemplo, el caso de Castilla y León, el Consejo Asesor estaba formado por 10 miembros, nombrados por la Junta de Castilla y León, nueve de ellos a propuesta de las Cortes y se designaban en función de la representatividad de cada grupo parlamentario. Entre las funciones del Consejo Asesor también se encontraba la elaboración de una memoria en la que recogía la situación de los medios de RTVE en esta comunidad y los acuerdos adoptados en el transcurso de sus reuniones. Estas reuniones se tenían que producir, al menos, una vez cada dos meses y una vez, como mínimo, cada seis meses tenía que elevar al Consejo de Administración de RTVE, a través del Delegado territorial, las recomendaciones que consideraban adecuadas respecto a la programación.

En el estudio del Estatuto de 1980 hay que llegar hasta la Disposición Adicional Segunda para encontrar la posibilidad de que RTVE ponga en funcionamiento un tercer canal regional:

En lo que respecta a televisión, RTVE en un principio articulará en la forma prevista en este Estatuto la programación específica destinada a cada nacionalidad o región de forma complementaria a la programación nacional que se emita por las dos cadenas existentes. Posteriormente extendida la cobertura técnica de ambas cadenas a todo el territorio español, el Gobierno, en los términos previstos en el artículo 2 del presente Estatuto, autorizará a RTVE a tomar las medidas necesarias para la puesta en funcionamiento de un tercer canal regional para el ámbito territorial de cada Comunidad Autónoma.

Parece que queda claro, que es potestad de RTVE, previa autorización del Gobierno quien puede poner en marcha un tercer canal autonómico, sin embargo al remitirse al artículo 2 (Capítulo I, Artículo $2^{\circ} .2$ ) del Estatuto, encontramos que:

El Gobierno podrá conceder a las Comunidades Autónomas, previa autorización por Ley de las Cortes Generales, la gestión directa de una canal de televisión de titularidad estatal que se cree específicamente para el ámbito territorial de cada Comunidad Autónoma.

Aquí tenemos que decir, que la regulación de los primeros terceros canales no se produce hasta que se aprueba la Ley 46/1983, Ley del Tercer Canal, para las televisiones autonómicas nacientes. Pero tenemos que añadir que como recoge Enrique Bustamante, RTVE no tuvo intención de establecer un canal regional hasta que, bajo el mandato de Pilar Miró se tomó la iniciativa de convertir TVE2 en un canal regional para competir con las televisiones autonómicas recientes y para conseguir los mayores ingresos de publicidad posible (Bustamante, 2013).

Como podemos ver, este Estatuto sienta las bases del funcionamiento, en Democracia, de los medios de comunicación de carácter público y estipula cuáles deben ser funciones al considerarse de servicio público. También especifica cómo debe ser la estructura territorial de RTVE pero el legislador no profundiza en ningún aspecto que concierna a las horas de programación que TVE tendría que realizar en cada territorio. De esta forma, la Dirección de TVE se convirtió en autónoma para decidir qué acciones debía seguir en cada caso. Al mismo tiempo, aunque los Consejos Asesores sí que elevaban informes, no siempre TVE tomó el compromiso de incrementar las ventanas de programación regional, al menos, en el caso de Castilla y León, salvo que existiese un convenio económico, sufragado por el Gobierno regional, para conseguir este objetivo.

\subsection{Ley 17/2006, de 5 de junio, de la radio y la televisión de titularidad estatal}

Esta Ley, aprobada durante la primera legislatura del Gobierno de José Luis Rodríguez Zapatero y se realizó una vez que el denominado Comité de Sabios realizó un informe en el que evaluó la situación de RTVE y cuáles debían ser sus herramientas de mejora para convertirse en un referente de calidad mediática. En el informe que presentó este Comité en el mes de febrero de 2005, los miembros del

\footnotetext{
${ }^{4}$ Capítulo II, Sección I Artículo V.1.a, en este punto se añade que las programaciones de RTVE deben impulsar el conocimiento de los valores constitucionales, promover el pluralismo, con respeto a las minorías, mediante el debate democrático, la información objetiva y plural y la libre expresión de opiniones, promover el respeto de la dignidad humana y de los derechos de los menores, la igualdad de sexos, la no discriminación por motivos de raza, ideología, religión o cualquier otra circunstancia social e impulsar la diversidad cultural y lingüística, entre otras cuestiones que conforman las características de servicio público y que se recogen en este artículo.

${ }^{5}$ El Consejo Asesor de RTVE en Castilla y León se creo mediante la Ley 1/1984, de 28 de junio, aunque, posteriormente, la Ley 2 /1989, de 10 de marzo, autorizó a la Junta de Castilla y León a refundir en un solo texto las disposiciones legales vigentes Reguladoras del Consejo Asesor de Radiotelevisión Española en Castilla y León. Este texto refundido se recoge en el Decreto Legislativo 1/1989, de 4 de mayo.
} 
mismo aseguran que es necesaria una reforma de estos medios de comunicación para responder así a las quejas de una parte de los consumidores; también se hace un requerimiento para regular la financiación de los medios públicos de comunicación y que éste sea viable.

\subsubsection{El Informe para la Reforma de los medios de comunicación de Titularidad del Estado}

El Informe que se presentó en el mes de febrero de 2005 analiza los aspectos de mejora de TVE y persigue que la nueva estructura organizativa de RTVE cumpla realmente con lo que los ciudadanos esperan de unos medios públicos de comunicación. Este documento también recoge cómo se deben regir los designios de RNE y de la Agencia EFE, como agencia de noticias dependiente del Estado. El texto presentado por los expertos también añade cuestiones relativas a la financiación de los medios públicos para que estos sean viables, nueva forma societaria y con qué órganos de control externos e internos deben contar para que sus funciones sean ejercidas a lo que el legislador y los ciudadanos esperan de ellos. Así, los sabios plantean la creación de los consejos de informativos que, más tarde, serán reflejados en la ley y que entrarán en funcionamiento unos meses después.

Este Informe recoge aspectos sobre cuáles deben ser las actividades y misiones de la radiotelevisión estatal y, concretamente, en el caso de TVE, cómo deben ser los dos canales. En este caso, se indica que TVE-1 debe conformarse como un canal generalista, de todos los géneros y para todos los públicos y debe constituirse como un referente básico del sector audiovisual (Informe para la Reforma de los medios de comunicación de titularidad del Estado, 2005). Respecto a La Dos, segundo canal de TVE, se pide que se convierta "en complementario del servicio público, orientado a la satisfacción de las demandas, gustos e intereses de las múltiples minorías existentes en la sociedad española" (Informe para la Reforma de los medios de comunicación de titularidad del Estado, 2005, p.99).

A la hora de valorar la estructura productiva y territorial de TVE, el Informe del Comité apuesta por una descentralización territorial que, además, favorezca la creación de industrias de la cultura el Informe para la Reforma de los medios de comunicación de titularidad del Estado (2005, p.105) :

(...) adquiere especial relevancia (dentro de un Estado plural y múltiple de lenguas y culturas), una estructura suficiente, diseminada en el conjunto del territorio, que no sólo sea capaz de alimentar de forma diversa y descentralizada las programaciones nacionales, sino que sostenga emisiones desconectadas específicas y sistematizadas. Unas emisiones que atiendan a las demandas regionales, siempre a favor de un papel vertebrador del conjunto del Estado. Igualmente, esas actividades deben ir fortaleciendo la descentralización de la industria audiovisual española, condición sine qua non para la desconcentración geográfica efectiva de las industrias de la cultura.

En este sentido, los expertos que realizaron el análisis de RTVE y posterior Informe indican que TVE debía conformar una nueva oferta de canales temáticos, para su emisión en los canales de TDT, pensados en misiones específicas de servicio público. Pero también señalan la importancia de la entra en vigor de un canal inter-regional que sea capaz de difundir en todo el territorio nacional la riqueza y la diversidad social y cultural de las comunidades autónomas y, para ello, asegura este Informe, es necesaria la aportación "intensiva" de los centros territoriales y en colaboración creciente con las televisiones autonómicas (Informe para la Reforma de los medios de comunicación de titularidad del Estado, 2005).

A la vista de los expuesto en estos párrafos parece claro que los responsables de este trabajo tenían claro que se debía reforzar el trabajo de los centros territoriales de TVE y se apuesta, en este sentido, por la creación de un canal en el que los contenidos sean específicamente creados por estas delegaciones, con aportaciones de las emisoras autonómicas con el objetivo de que el espectador pueda conocer las características propias de cada comunidad autónoma.

Una vez presentado el Informe, comenzó el trámite legislativo para la aprobación el 5 de junio de esta Ley que iba a reorganizar los medios de comunicación dependientes del Estado y que contaban con la misma regulación básica, salvo pequeñas modificaciones, desde el Estatuto de RTVE de 1980. En la Exposición de Motivos de esta Ley ya se avanza que el objetivo de la misma es dotar a la radio y la televisión dependientes del Estado de un régimen jurídico que garantice su independencia, neutralidad y objetividad. A esto añade el legislador que servirá para establecer las estructuras organizativas adecuadas así como un modelo de financiación que les permita cumplir con su función de servicio público.

La Ley también define la función de servicio público estatal de radio y televisión, con una programación de calidad y con el fomento de la producción española y europea. En el articulado se recogen cuestiones sobre la composición del Consejo de Administración y cuáles deben ser los principios que rijan la producción y programación de los contenidos de la radio y la televisión públicas así como la garantía de acceso de los grupos sociales y políticos significativos.

En el articulado de esta ley encontramos cuestiones relacionadas con la cobertura universal, alcanzar la mayor cobertura dentro del territorio nacional de los servicios de difusión de radio y televisión. También se indica en el artículo 3.2 en cuando al desarrollo del su función de servicio público que la Corporación RTVE deberá promover la cohesión territorial, la pluralidad y la diversidad lingüística y cultural de España (Artículo 3.2 e). Ley 17/2006). Dentro del ámbito de estudio que nos interesa en este caso y referido a los centros territoriales de TVE, tenemos que llegar al artículo 7.4 de la Ley 17/ 2006 para encontrar que:

La Corporación RTVE contará con la estructura territorial necesaria para atender la adecuada prestación de sus funciones de servicio público, proveer de contenidos regionalizados a la realidad estatal, contribuir al desarrollo de la cohesión interterritorial, atendiendo al hecho insular y las condiciones de las regiones ultraperiféricas. Las desconexiones se harán en la lengua propia de las CC.AA.

Esta Ley recoge aspectos novedosos que, hasta ese momento, no se habían visto ligados a las televisiones públicas en España. En este caso, dentro de los Principios de producción y programación, se indica que la Corporación RTVE y sus sociedades deberán ajustarse en 
estas cuestiones al cumplimiento de sus funciones de servicio público. Para ello, el contrato-programa, de acuerdo con las líneas del mandato marco, recogerá los objetivos y obligaciones específicas que deben cumplir los distintos canales de radio y televisión (Artículo 21.1 y Artículo 25.2 Ley 17/2006). El mandato-marco lo aprobarán las Cortes Generales concretando los objetivos de servicio público y tendrá una vigencia de nueve años. En concreto, el contrato-programa, con una vigencia de tres años, determina las aportaciones anuales, con cargo a los Presupuestos del Estado para cada ejercicio. Este contrato-programa los firmarán el Gobierno y la Corporación y asumirá los objetivos concretos que se tendrán que desarrollar en ese ejercicio en la función de servicio público (García Castillejo, 2014).

En cuanto a la financiación, esta Ley recoge que el sistema de ingresos de la nueva Corporación ha de ser mixto, con financiación del Estado para compensar el desarrollo de las funciones de servicio público y por los ingresos recogidos de su participación en el mercado publicitario. Sin embargo, como veremos en el siguiente apartado, este sistema de financiación se modificó pocos años después.

\subsubsection{Primer Mandato-Marco a la Corporación RTVE}

Una vez constituida la Corporación RTVE con fecha 12 de septiembre de 2006, se elaboró el primer mandato-marco con una vigencia de nueve años que contarían desde su entrada en vigor, el 1 de enero del año 2008. Este mandato se probó en el Pleno del Congreso de los Diputados el 11 de diciembre de 2007 y en el Pleno del Senado en la sesión del 12 de diciembre de 2007. En el articulado de este primer mandato se recoge que la Corporación RTVE tiene que cumplir los objetivos de servicio público que ya se recogen en la Ley 17/2006. A esto se añade que deberá defender y difundir los valores constitucionales y contribuir a la vertebración y a la cohesión territorial (Artículo 9). Además, la Corporación, se recuerda en el Artículo 10, debe ser un sujeto activo en la defensa de los valores contemplados en el artículo 20 de la Constitución Española y se debe regir por los principios de independencia, neutralidad, pluralismo, imparcialidad y rigor.

Dentro del mandato marco también se recogen cuestiones relacionadas con la promoción de los intereses españoles en el panorama internacional teniendo que hacer llegar la realidad actual de España a los cinco continentes. Respecto a las emisiones territoriales, éstas se deben hacer en la lengua cooficial en función de la realidad lingüística de cada Comunidad Autónoma.

En el Capítulo III de este mandato-marco se marcan los objetivos programáticos de la función de servicio público que pasan por producir y difundir contenidos de calidad en todos los géneros y, sobre todo, en los de carácter informativo, educativo, cultural y divulgativo. Sobre la programación informativa, en el Artículo 23 se pide a la Corporación que dé prioridad a la información que se "debe constituir en eje fundamental de su oferta y un espacio de debate público" para favorecer la reflexión, el conocimiento de la realidad, la actitud crítica y la participación ciudadana. Sobre los programas de entretenimiento, en este capítulo se pide que sean de calidad y no podrán entrar en contradicción con los principios de servicio público.

En el Capítulo IV se marcan las líneas estratégicas de contenidos, emisión y producción. En el Artículo 30, respecto a la oferta televisiva, se indica que los canales generalistas ofrecerán la posibilidad de desconexiones para los circuitos territoriales que "garantizan el acceso a los contenidos de proximidad", pero en este caso, no se especifica la frecuencia o duración de estas desconexiones. Hay que llegar hasta el Artículo 33.b para encontrar lo siguiente:

Las emisiones con cobertura específicamente territorial se concretarán a través de desconexiones que, en función de la estrategia general de la programación prevista por cada una de las Sociedades Mercantiles de RNE y TVE, emitirán únicamente contenidos de proximidad contemplados en los artículos 29 y 30 de este Mandato-marco. Lo porcentajes de emisión, así como los canales y recursos destinados a la estructura territorial se fijarán en cada Contrato-programa6.

Pese a las estipulaciones recogidas en la Ley 17/2006 y a lo que se exige en este Mandato-marco que entró en vigor en el mes de enero de 2008, no se ha firmado ningún Contrato-programa por parte de ningún gobierno 7 . A esto hay que añadir que en la fiscalización de las cuentas de la Corporación RTVE del ejercicio 2010-2011, el Tribunal de Cuentas exige a la Corporación y al Gobierno que se firme el Contrato-programa para garantizar la financiación de la misma ${ }^{8}$. Respecto a la estructura y dimensionamiento de la Corporación RTVE mantendrá su estructura territorial, tanto de radio como de televisión, con capacidad para producir y emitir contenidos informativos. Para realizar este cometido, la Corporación se dotará de sistemas tecnológicos de máxima autonomía y eficacia de recursos. TVE debe mantener su presencia en todas las Comunidades Autónomas del Estado Español con una unidad de emisión y producción de noticias (Artículo 41).

Como vemos en este primer Mandato-marco sólo se marcan las líneas generales de lo que debe ser el funcionamiento de la Corporación RTVE para cumplir con las funciones asignadas de servicio público. Sin embargo, no contar con la firma del Contrato-programa no ha permitido concretar aspectos tales como la programación de los Centros Territoriales.

\subsection{Ley 8/2009, de 28 de agosto, de financiación de la Corporación de Radio y Televisión Española}

Podemos indicar que esta Ley es un reglamento complementario de la anterior de 2006 y viene a fijar cómo debe ser la financiación de la nueva Corporación. En el Preámbulo de esta Ley que se publicó en el BOE el 31 de agosto, el legislador viene a justificarla indicando que

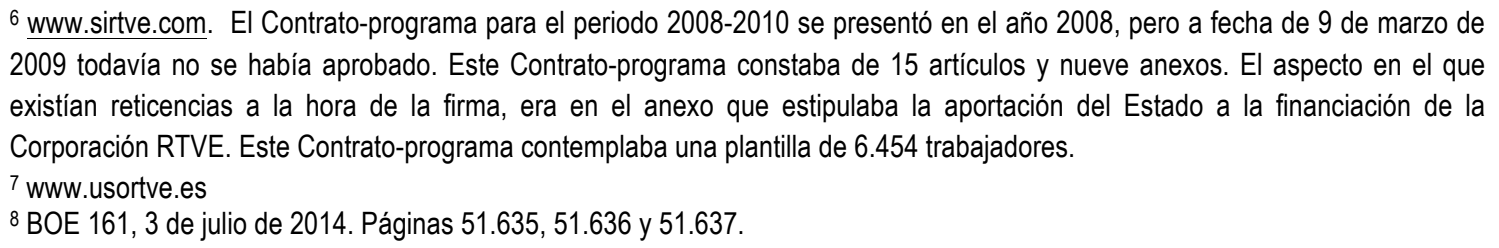

${ }^{6}$ www.sirtve.com. El Contrato-programa para el periodo 2008-2010 se presentó en el año 2008, pero a fecha de 9 de marzo de 2009 todavía no se había aprobado. Este Contrato-programa constaba de 15 artículos y nueve anexos. El aspecto en el que existían reticencias a la hora de la firma, era en el anexo que estipulaba la aportación del Estado a la financiación de la Corporación RTVE. Este Contrato-programa contemplaba una plantilla de 6.454 trabajadores.

${ }^{8}$ BOE 161, 3 de julio de 2014. Páginas 51.635, 51.636 y 51.637. 
la nueva norma se ajusta a la Directiva Europea 2007/65/CE del Parlamento Europeo y del Consejo de 11 de diciembre que ya limitaba los tiempos de emisión de publicidad en la Corporación RTVE. El legislador argumenta esta Ley indicando que, en la situación del año 2009, lo más aconsejable es acelerar el proceso de cambio del modelo de financiación de RTVE para renunciar definitivamente, y de forma inmediata, a los ingresos publicitarios. De esta manera, se pasa a un sistema de financiación único basado en ingresos públicos.

Esta Ley vincula la financiación de RTVE no solo a las aportaciones que realice el Estado a través de sus Presupuestos Generales sino que también quiere que los beneficiarios de la eliminación de la publicidad participen, en un porcentaje determinado, en la financiación de la radio y la televisión públicas. De esta manera, se contempla un porcentaje sobre los ingresos de los operadores de televisión comercial en abierto del 3\%; del 1,5\% para los operadores de televisión de pago y del $0,9 \%$ para los de telecomunicaciones. El legislador en el Preámbulo de esta Ley indica que ésta es una fórmula que ya se usa en otros países del entorno europeo y que los porcentajes de participación financiera fijados para los diferentes operadores se han realizado una vez elevada una consulta al Consejo de Estado.

\subsection{Ley 7/2010, de 31 de marzo, General de la Comunicación Audiovisual}

Esta Ley, publicada en el BOE de 1 de abril de 2010, venía a cumplir las demandas que los operadores habían realizado durante los últimos años. Además, supuso la derogación de leyes anteriores como las que regulaban el tercer canal, la televisión privada o la del impulso de la TDT (Zallo R. 2011, p: 53). Esta ley se presenta como una norma básica, fundamental tanto para el sector privado como para

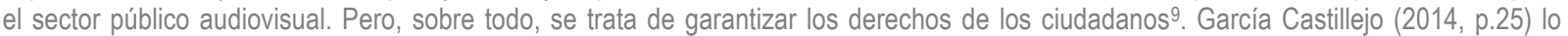
analiza de la siguiente forma:

La Ley General crea un marco enfocado a la garantía de los derechos de los ciudadanos a recibir comunicación audiovisual en condiciones de pluralismo cultural y lingüístico (...) así como a exigir ante las autoridades la adecuación de los contenidos al ordenamiento constitucional español vigente.

Así, esta Ley recoge cuáles deben ser las obligaciones de los prestadores del servicios de comunicación audiovisual en relación con los menores y las personas con discapacidad que merecen, según estipula el legislador, una atención y protección especiales. Pero esta Ley también recoge los derechos de los prestadores de los servicios de comunicación audiovisual ${ }^{10}$. En este sentido, el profesor Ramón Zallo recuerda que para los prestadores privados desaparece el concepto de servicio público y pasa a considerarse de "interés general"11. Estos prestadores tendrán derecho a ofrecer el servicio en condiciones de libertad respecto a la selección de los contenidos, su línea editorial y la emisión de los canales. Además, aplicando los criterios legislativos europeos, esta Ley estipula que se pase de las concesiones por periodos de 10 años, a periodos de licencia de quince años, así como se establece la renovación automática del mismo si se cumplen los requisitos determinados (García Castillejo, 2014).

A nuestro juicio, la ampliación del tiempo de concesión de licencias y la posibilidad de renovación automática de las mismas es una garantía para los operadores privados que pueden establecer planes de desarrollo y de negocio más amplios, sin estar pendientes de la caducidad o medio plazo de la licencia de explotación de una determinada frecuencia. Por lo que podemos indicar que esta Ley ofrece unas mayores garantías a los operadores privados de televisión al facilitar el desarrollo de su volumen de negocio que permite, incluso, la fusión entre ellos, como hemos visto en el capítulo anterior. Otro aspecto importante de esta Ley lo encontramos en lo referido a lo que se denomina alfabetización mediática de los ciudadanos y que se recoge en el artículo 6.4 de este nueva Ley: "Los poderes públicos y los prestadores del servicio de comunicación audiovisual deben contribuir a la alfabetización mediática de los ciudadanos".

Respecto a los prestadores públicos del servicio de comunicación audiovisual su regulación en esta Ley aparece en el Capítulo IV, entre los artículos 40 al 43. Aquí se recoge que el servicio público de comunicación audiovisual es un servicio esencial de interés económico general que tiene como misión difundir contenidos que fomenten los principios y valores constitucionales, contribuir a la formación de una opinión pública plural, dar a conocer la diversidad cultural y lingüística de España y difundir el conocimiento de las artes, con especial incidencia en el fomento de la cultura audiovisual (Artículo 40.1 Ley 7/2010).

De esta manera, el legislador entiende que el servicio público de comunicación audiovisual tiene por objeto la producción, edición y difusión de un conjunto de canales de radio, televisión y servicios de información en línea con programaciones equilibradas y diversas para todo tipo de público. Respecto al control de la función del servicio público audiovisual, en el articulado de la Ley se recoge que, desde la entrada en vigor de esta norma y en un plazo de 180 días, el Gobierno tendrá que suscribir con la Corporación RTVE el contrato programa en el que se debe detallar la oferta televisiva y que tiene que recoger las obligaciones de servicio público que se estipulan en la Ley 17/2006 que ya hemos explicado anteriormente y cumplir los aspectos de la Ley 8/2009 sobre Financiación de la Corporación RTVE. Así, también se contempla que el prestador de servicio público de comunicación audiovisual deberá contar con la organización y estructura suficiente y

\footnotetext{
${ }^{9}$ Los Derechos del Público se recogen en el Título II: Normativa Básica para la Comunicación Audiovisual, en el Capítulo I: los derechos del público, entre los artículos 4 y 9 , entre los que se recogen, además de los mencionados anteriormente, aspectos relacionados con el derecho a la participación y control de los contenidos audiovisuales.

10 Los derechos de los prestadores del servicio público de comunicación audiovisual se recogen en el Capítulo II, del Título II de esta Ley 7/2010 y entre los que se incluyen también, el derecho a organizar comunicaciones comerciales, el derecho a la autorregulación del prestador, el derecho al patrocinio y al emplazamiento de productos. Sin embargo queda prohibida la comunicación ilícita y toda aquella comunicación comercial que vulnere la dignidad humana, así como la comunicación comercial encubierta, entre otras cuestiones comerciales (artículo 18, Ley 7/2010).

${ }^{11}$ El servicio de comunicación audiovisual se considera de interés general. Antes de esta Ley, la televisión de gestión privada estaba sometida a obligaciones de servicio público definidas por las leyes o las que se derivaban de la obtención de la licencia (Zallo, R. en El nuevo escenario mediático, Campos Freire, F. (coord..). Zamora, 2011, Comunicación Social, ediciones y publicaciones.
} 
Respecto a los límites de los prestadores públicos el Artículo 42.1 indica que estos no pueden participar en el capital social de prestadores privados del servicio de comunicación audiovisual. Además, sobre la dirección editorial de los prestadores públicos la Ley indica que estos criterios editoriales se deberán elaborar por un órgano compuesto por miembros que reflejen el pluralismo político y social de su ámbito de cobertura (Artículo 42.3 Ley 7/2010).

\subsection{Real Decreto-ley 15/2012, de 20 de abril}

Este Real Decreto-ley de modificación del régimen de administración de la Corporación RTVE supuso la reducción del número de miembros del Consejo de Administración de la Corporación RTVE que pasó de tener 12 miembros a nueve. Este Real Decreto-ley afecta básicamente a la organización del Consejo de Administración de la Corporación de RTVE, no tanto a su consideración como prestador público de servicios de comunicación audiovisual. Esta modificación también supuso que cambios en la composición del Consejo Asesor y del Presidente de la Corporación RTVE. De esta manera, como explica Ángel García Castillejo, se permite que si no se logra la mayoría de dos tercios para la elección de los miembros del Consejo de Administración en la Cámara correspondiente, la votación se pueda repetir a las 24 horas; en este caso, cada Cámara elegirá, por mayoría absoluta, a los consejeros que les corresponda. Para la elección del Presidente de la Corporación se utiliza un sistema similar, de al forma que si no se consigue la mayoría de dos tercios en el Congreso de los Diputados, la designación pueda llevarse a cabo por mayoría absoluta, una vez transcurridas 24 horas desde la votación anterior.

\subsection{Comisión Mixta de Control de RTVE}

Desde que se aprobó el Estatuto de RTVE en el año 1980, RTVE ha estado sometida al control parlamentario. En el caso del Estatuto de 1980 encontramos en la sección V, artículo 26, las cuestiones que se refieren al ejercicio de este control. Así se explica que se constituirá una Comisión Parlamentaria del Congreso de los Diputados de conformidad con lo que disponga el Reglamento de la Cámara. Esta Comisión tiene como función el control de la actuación del Ente Público RTVE y sus Sociedades para velar por el cumplimiento de la función de servicio público. Además, RTVE entre sus compromisos con la Comisión tenía la presentación, de forma anual, de una memoria o informe sobre la ejecución de la función de servicio público en la que también se tendrían que incluir cuestiones relativas a programación, servicios y emisiones. En el articulado que recoge el control de RTVE en el Parlamento se indica que ésta tendrá que presentar la información adecuada a la comisión que se cree en el Parlamento cada vez que se solicite.

Por su parte, en la Ley 17/2006 que ya hemos expuesto en este texto, hay que llegar al Capítulo VI para encontrar los artículos correspondientes al control externo ${ }^{12}$ de la nueva Corporación RTVE. Así, en el Artículo 39, respecto al Control del Parlamento se estipula que: "Las Cortes Generales ejercerán el control parlamentario sobre la actuación de la Corporación y sus Sociedades". En este control se tiene que velar por el cumplimiento de las funciones de servicio público, tal y como se recogía también en el Estatuto de 1980. También se incluye la obligación que tiene que cumplir la Corporación de remitir un informe anual a las Cortes Generales sobre la ejecución del Contrato-programa y del Mandato-marco.

Esta Comisión cuenta con representantes de todos los partidos políticos del arco parlamentario tanto del Congreso de los Diputados como del Senado. Ante esta Comisión la Corporación RTVE no sólo presenta sus informes anuales sino que también el Presidente de la Corporación comparece para responder a las preguntar efectuadas por los miembros de esta Comisión. A lo largo de las diferentes legislaturas en las que esta Comisión ha estado controlando el funcionamiento de RTVE, los miembros de la misma han realizado múltiples preguntas relacionadas con todos os aspectos del funcionamiento de RTVE. Entre los objetivos de las preguntas parlamentarias se han encontrado los índices de audiencia de los distintos espacios de TVE, las acusaciones de manipulación informativa en distintas etapas, la contratación de diferentes profesionales y también, la situación de los Centros Territoriales de TVE.

En el transcurso de las distintas legislaturas, las cuestiones relativas a estas delegaciones regionales sí han ocupado tiempo y espacio en la Comisión al igual que otros centros de trabajo de RTVE. Estas inquietudes de los parlamentarios sobre el trabajo desempeñado en los Centros Territoriales ha sido más importante en los momentos en los que existían dudas sobre el mantenimiento o no de los mismos. También se han elevado preguntas antes y después del desarrollo de las citas electorales con el objetivo de conocer cómo se había realizado esa cobertura.

\section{Conclusiones}

Después del análisis realizado podemos indicar que existen dos grandes normas sobre las que se han regido los designios de RTVE. En primer lugar, encontramos el Estatuto de RTVE y, posteriormente, la Ley 17/2006 cuya intención era crear unos medios públicos de calidad y que cumplieran rigurosamente con las funciones encomendadas de servicio público. Sin embargo, en ninguno de los dos textos legislativos encontramos cuestiones que desarrollen, con detalle, cómo debe ser la estructura territorial de RTVE, en concreto de TVE, para el desarrollo de estos fines.

Si bien es cierto, que en los artículos 13, 14 y 15 del Estatuto de 1980 se indica cómo debe ser la estructura territorial de RTVE, no se especifica qué aspectos concretos debe tener esta estructura territorial. Con estos nos referimos a que no incluye qué tipo de

\footnotetext{
${ }^{12}$ Este Capítulo VI también recoge, en el Artículo 40, la relación entre la Corporación RTVE y la autoridad audiovisual. En este aspecto se indica que es esta autoridad audiovisual quien tiene que supervisar que la Corporación cumple con la misión de servicio público. En el Artículo 41, se explica que corresponde al Tribunal de Cuentas el control externo de la Corporación y el de las sociedades en que participe, directa e indirectamente, de forma mayoritaria.
} 
programación, en cuanto a espacios de información y entretenimiento, ha de seguirse en estos territorios o la determinación de cuánta duración han de tener las desconexiones para esos territorios. En este sentido, se deja al cargo de la Dirección general de RTVE la determinación cómo deben darse estas circunstancias por lo que no parece que exista intención de que la programación territorial sea amplia.

Respecto a lo que sucede en la Ley 17/2006 nos encontramos con una indefinición similar cuando en el Capítulo I, en el Artículo 7 sobre la estructura de la Corporación RTVE, en el apartado 4, se indica que esta Corporación deberá contar con la estructura necesaria a nivel territorial para el cumplimiento de las funciones de servicio público. Al igual que sucede con el texto legislativo anterior, tampoco se indica qué tipo de programación, en desconexión territorial se debe realizar en cada uno de los territorios autonómicos, ni la duración de estas desconexiones.

Si ahondamos un poco más, vemos que en el Mandato-marco que entró en vigor el 1 de enero de 2008 se deja a las cuestiones que se recojan en el Contrato-programa, de vigencia trianual todo lo referido a la programación territorial. Aquí nos encontramos que, con fecha, de mayo de 2014 no se había firmado este Contrato-programa por lo que las desconexiones territoriales seguían las directrices marcadas por la dirección de TVE y la Presidencia de la Corporación RTVE y no las que se pudieran contemplar en con Contrato-programa.

Es entonces cuando podemos deducir que, en el marco legislativo, no ha existido una concreción clara, por parte del legislador, para determinar de una forma nítida cuáles deberían ser las directrices concretas de las desconexiones territoriales de TVE y cómo se debían organizar las mismas así como su extensión. Esto ha supuesto un inconveniente importante para estas delegaciones ya que no han podido desarrollar producciones de servicio público para sus ámbitos geográficos, limitando así la presencia de la televisión pública nacional en las distintas comunidades autónomas.

\section{Bibliografía}

Abad Alcalá, L. (1999). El servicio público de televisión ante el siglo XXI. Madrid. Dykinson

Bustamante, E. (2013). Historia de la radio y la televisión en España. Una asignatura pendiente de la democracia. Barcelona. Gedisa

Bustamante, E. (2004). La televisión económica. Financiación, estrategias y mercados. Barcelona. Gedisa

García Castillejo, Á. (2014). La televisión en España, Marco legal. Barcelona. UOC

González Encinar, J.J. (1996). La televisión pública en la Unión Europea. Madrid. McGraw-Hill

Zallo, R. (2011). Políticas de comunicación audiovisual en (y para después de) la crisis económica en España. En Campos Freire, F. (coord.) El nuevo escenario mediático (pp. 49-80). Zamora, Sevilla. Comunicación Social ediciones y publicaciones

C. E. Aprobada el 6 de diciembre de 1978

Estatuto de Radio y Televisión 1980. BOE n 11, de 12 de enero de 1980, págs.. 844-848

Ley $17 / 2006$ de 5 de junio. BOE n⿳0 134, de 6 de junio de 2006, págs. 21207-21218

Ley 8/2009, de 28 de agosto, de financiación de la Corporación de Radio y Televisión Española. BOE n² 210, de lunes 31 de agosto de 2009, págs.74003-74015

Ley 7/2010, de 31 de marzo, General de la Comunicación Audiovisual. BOE nº 79, de 1 de abril de 2010

Real Decreto-ley 15/2012, de 20 de abril. BOE n 96, de 21 de abril de 2012, págs.. 30985-30991

www.abc.es

www.rtve.es

www.elpais.es

www.sirtve.es

\section{Cómo citar este artículo en bibliografías - How to cite this article in bibliographies / references:}

PÉREZ-RUIZ, M. (2016): “TVE, su legislación y su afectación en los Centros Territoriales"”. En Revista de la Asociación Española de Investigación de la Comunicación, vol. 3, número 6, pp. 43-50. 